\title{
The Curvature Property of a Linear Dynamical System
}

\author{
Tuna Bayrakdar $^{1 *}$, Zahide Ok Bayrakdar ${ }^{2}$ \\ 1* Trakya University, Faculty of Sciences, Departmant of Mathematics, Edirne, Turkey, (ORCID: 0000-0001-8777-5842), tunabayraktar@gmail.com \\ ${ }^{2}$ Ege University, Faculty of Sciences, Departmant of Physics, İzmir, Turkey, (ORCID: 0000-0002-2187-2102), zahideok@gmail.com
}

(1st International Conference on Applied Engineering and Natural Sciences ICAENS 2021, November 1-3, 2021)

(DOI: 10.31590/ejosat.1014593)

ATIF/REFERENCE: Bayrakdar, T. \& Ok Bayrakdar, Z. (2021). The Curvature Property of a Linear Dynamical System. European Journal of Science and Technology, (28), 1288-1290.

\begin{abstract}
In this work a two-dimensional smooth autonomous dynamical system is regarded as a three-dimensional Riemannian manifold and it is shown that the scalar curvature of a linear dynamical system $\mathrm{d} x / \mathrm{d} t=a x+b y, \mathrm{~d} y / \mathrm{d} t=c x+d y$ is non-positive. The manifold is scalar-flat iff $b=-c$ and $a=d=0$.
\end{abstract}

Keywords: Linear dynamical systems, Riemann curvature tensor, scalar curvature

\section{Bir Lineer Dinamik Sistemin Ĕ̆rilik Özelliği}

$\ddot{O} \mathbf{z}$

Bu çalışmada iki-boyutlu, düzgün, otonom bir dinamik sistem üç-boyutlu bir Riemann manifoldu olarak değerlendirilmiş ve bir $\mathrm{d} x / \mathrm{d} t=a x+b y, \mathrm{~d} y / \mathrm{d} t=c x+d y$ lineer dinamik sisteminin skaler eğriliğinin pozitif olmadığ gösterilmiştir. Manifold skalerdüzdür ancak ve ancak $b=-c$ ve $a=d=0$.

Anahtar Kelimeler: Lineer dinamik sistemler, Riemann eğrilik tensörü, skaler eğrilik.

\footnotetext{
* Corresponding Author: tunabayraktar@gmail.com
} 


\section{Introduction}

A smooth autonomous dynamical system (SADS) on a twodimensional manifold $N=(D ; x, y)$, where $D$ is a connected open set in $\mathbb{R}^{2}$ endowed with coordinates $(x, y)$, is given by a system of first order ordinary differential equations

$$
\begin{aligned}
& \frac{\mathrm{d} x}{\mathrm{~d} t}=f(x, y) \\
& \frac{\mathrm{d} y}{\mathrm{~d} t}=g(x, y)
\end{aligned}
$$

such that $f$ and $g$ are smooth functions on $N$. The system (1) defines a smooth vector field

$$
\xi=f(x, y) \partial_{x}+g(x, y) \partial_{y}
$$

on $N$ which is a smooth section of the tangent bundle $T N$, i.e. is a mapping $\xi: N \rightarrow T N$ defined to be

$$
\xi(x, y)=(x, y, \dot{x}=f(x, y), \dot{y}=g(x, y)) .
$$

Since the rank of $\mathrm{d} \xi$, the differential of $\xi$, equals 2 on $N$, a SADS may be regarded as a surface in $T N$.

In this work, to capture all the information about the dynamics, we identify a SADS as a three-dimensional manifold $M=I \times N$ with local coordinates $(t, x, y)$. Here $t$ is a coordinate function on an open interval $I \subset \mathbb{R}$ and stands for the time coordinate. This consideration gives rise to define a SADS as a submanifold of the first-order jet bundle $J^{1}(\mathbb{R}, N)$ of maps $\mathbb{R} \rightarrow N[1,2]$.

This paper is devoted to the investigation of the curvature property of a linear system

$$
\begin{aligned}
& \frac{\mathrm{d} x}{\mathrm{~d} t}=a x+b y \\
& \frac{\mathrm{d} y}{\mathrm{~d} t}=c x+d y,
\end{aligned}
$$

where $a, b, c, d \in \mathbb{R}$, within the context of Riemannian geometry. By means of the method of moving frames we evaluate the connection 1-form and the curvature 2-form of the Levi-Civita connection in $T M$ compatible with the Riemannian metric which is defined by the sum of squares of the one-forms

$$
\begin{aligned}
\omega^{1} & =\mathrm{d} t \\
\omega^{2} & =\mathrm{d} x-(a x+b y) \mathrm{d} t \\
\omega^{3} & =\mathrm{d} y-(c x+d y) \mathrm{d} t
\end{aligned}
$$

and show that the scalar curvature of the connection is nonpositive and the curvature vanishes if and only if $b=-c$ and $a=d=0$.

\section{Riemannian structure and the curvature}

The solutions of (3) are identified with the solutions of the Pfaffian system

$$
\omega^{2}=0, \quad \omega^{3}=0
$$

such that on a solution curve we have $\omega^{1} \neq 0$. The column vector $\omega=\left(\omega^{1}, \omega^{2}, \omega^{3}\right)^{t}$, where ${ }^{t}$ denotes the transposition, defines a coframe on $M$ which is dual to the frame of the vector fields

$$
e_{1}=\frac{\partial}{\partial t}+f \frac{\partial}{\partial x}+g \frac{\partial}{\partial y}, \quad e_{2}=\frac{\partial}{\partial x}, \quad e_{3}=\frac{\partial}{\partial y} .
$$

If we introduce the Riemannian metric on $M$

$$
d s^{2}=\sum_{i} \omega^{i} \otimes \omega^{i},
$$

then $M$ becomes a Riemannian manifold and the frame $e=$ $\left(e_{1}, e_{2}, e_{3}\right)$ forms an orthonormal frame. Let $\nabla$ be the connection compatible with the Riemannian metric (4). The structure equations for the coframe $\omega$ are given by

$$
\begin{aligned}
\mathrm{d} \omega^{1} & =0 \\
\mathrm{~d} \omega^{2} & =a \omega^{1} \wedge \omega^{2}+b \omega^{1} \wedge \omega^{3} \\
\mathrm{~d} \omega^{3} & =c \omega^{1} \wedge \omega^{2}+d \omega^{1} \wedge \omega^{3} .
\end{aligned}
$$

As it is considered in [4], the $\mathfrak{s D}(3, \mathbb{R})$-valued connection form is obtained by solving the system of equations

$$
d \omega^{i}=-\theta_{j}^{i} \wedge \omega^{j}, \quad \theta_{j}^{i}=-\theta_{i}^{j} .
$$

The unique 1-form

$$
\theta=\left(\begin{array}{lll}
0 & \theta_{2}^{1} & \theta_{3}^{1} \\
-\theta_{2}^{1} & 0 & \theta_{3}^{2} \\
-\theta_{3}^{1} & -\theta_{3}^{2} & 0
\end{array}\right)
$$

satisfying the structure equations is obtained by the Cartan's Lemma, where

$$
\begin{gathered}
\theta_{2}^{1}=-a \omega^{2}-\frac{1}{2}(b+c) \omega^{3} \\
\theta_{3}^{1}=-\frac{1}{2}(b+c) \omega^{2}-d \omega^{3} \\
\theta_{3}^{2}=-\frac{1}{2}(b-c) \omega^{1} .
\end{gathered}
$$

The 1 -form $\theta$ is called $\mathfrak{s D}(3, \mathbb{R})$-valued connection form of the Levi-Civita connection.

The curvature 2-form of the Levi-Civita connection is defined by the anti-symmetric matrix of two-forms:

$$
\Omega_{j}^{i}=\mathrm{d} \theta_{j}^{i}+\sum_{k} \theta_{k}^{i} \wedge \theta_{j}^{k}, \quad \Omega_{j}^{i}=-\Omega_{i}^{j} .
$$

In terms of $\omega^{i}$ s, the components of the Riemannian curvature tensor are determined by

$$
\Omega_{j}^{i}=\sum_{k<l} R_{j k l}^{i} \omega^{k} \wedge \omega^{l} .
$$

For the details on a connection in an arbitrary vector bundle we refer to [3]. By a direct calculation we obtain

$$
\begin{aligned}
\Omega_{2}^{1} & =-\frac{1}{2}\left(2 a^{2}+\frac{3}{2} c^{2}+b c-\frac{1}{2} b^{2}\right) \omega^{1} \wedge \omega^{2} \\
& -(a b+c d) \omega^{1} \wedge \omega^{3} \\
\Omega_{3}^{1} & =-(a b+c d) \omega^{1} \wedge \omega^{2} \\
& -\frac{1}{2}\left(\frac{3}{2} b^{2}+b c+2 d^{2}-\frac{1}{2} c^{2}\right) \omega^{1} \wedge \omega^{3}
\end{aligned}
$$


$\Omega_{3}^{2}=\frac{1}{2}\left(\frac{1}{2}(b+c)^{2}-2 a d\right) \omega^{2} \wedge \omega^{3}$.

The independent nonzero components of the Riemann curvature tensor are

$$
\begin{aligned}
& R_{212}^{1}=-\frac{1}{2}\left(2 a^{2}+c(b+c)-\frac{1}{2}\left(b^{2}-c^{2}\right)\right) \\
& R_{213}^{1}=-(a b+c d) \\
& R_{313}^{1}=-\frac{1}{2}\left(b(b+c)+2 d^{2}+\frac{1}{2}\left(b^{2}-c^{2}\right)\right) \\
& R_{323}^{2}=\frac{1}{2}\left(\frac{1}{2}(b+c)^{2}-2 a d\right) .
\end{aligned}
$$

At a point, the sectional curvatures of the two dimensional subspaces spanned by $\left(\boldsymbol{e}_{1}, \boldsymbol{e}_{2}\right),\left(\boldsymbol{e}_{1}, \boldsymbol{e}_{3}\right)$ and $\left(\boldsymbol{e}_{2}, \boldsymbol{e}_{3}\right)$ are given respectively by $R_{212}^{1}, R_{313}^{1}$, and $R_{323}^{2}$. The scalar curvature is defined by the trace $\boldsymbol{R}=\sum_{i, j} \boldsymbol{R}_{j i j}^{i}$ and is found to be $\boldsymbol{R}=$ $\mathbf{2}\left(\boldsymbol{R}_{212}^{\mathbf{1}}+\boldsymbol{R}_{\mathbf{3 1 3}}^{\mathbf{1}}+\boldsymbol{R}_{\mathbf{3 2 3}}^{\mathbf{2}}\right)$. Note that the scalar curvature is an invariant, that is, it does not depend on the choice of an orthonormal frame. It follows that

$$
\begin{gathered}
R=-\left(2 a^{2}+\frac{3}{2} c^{2}+b c-\frac{1}{2} b^{2}+\frac{3}{2} b^{2}+b c+2 d^{2}-\frac{1}{2} c^{2}\right. \\
\left.-\frac{1}{2} b^{2}-\frac{1}{2} c^{2}-b c+2 a d\right) .
\end{gathered}
$$

By arranging the terms we obtain

$$
2 R=-\left((b+c)^{2}+(2 a+d)^{2}+3 d^{2}\right) .
$$

As a result we have the following:

Theorem 1 The Riemannian manifold corresponding to a linear system

$$
\begin{aligned}
& \frac{\mathrm{d} x}{\mathrm{~d} t}=a x+b y \\
& \frac{\mathrm{d} y}{\mathrm{~d} t}=c x+d y
\end{aligned}
$$

has non-positive scalar curvature. The scalar curvature vanishes if and only if $b=-c$ and $a=d=0$.

We say that the Riemannian manifold is flat iff the curvature tensor identically vanishes. Substituting $b=-c$ and $a=d=0$ into the components of the Riemannian curvature tensor yields the following:

Corollary 2 The Riemannian manifold corresponding to a linear system

$$
\begin{aligned}
& \frac{\mathrm{d} x}{\mathrm{~d} t}=\lambda y \\
& \frac{\mathrm{d} y}{\mathrm{~d} t}=-\lambda x,
\end{aligned}
$$

\section{References}

[1]Vassiliou, P. J. (2000). Introduction: Geometric approaches to differential equations. In Vassiliou P. J. and Lisle I. G. (Eds.), Geometric approaches to differential equations (pp. 1-15). Australian Mathematical Society Lecture Series. 15, Cambridge University Press, Cambridge UK.

[2]Saunders, D. J. (1989). The Geometry of Jet Bundles. Cambridge University Press, Cambridge UK.

[3] Morita, S. (2001). Geometry of Differential Forms. American Mathematical Society, Providence, RI, USA.

[4]Ok Bayrakdar, Z., Bayrakdar, T. (2019). A geometric description for simple and damped harmonic oscillators, Turk J Math, 43: 2540 - 2548.

where $\lambda \in \mathbb{R}$, is flat. 